\title{
Repair of Peroneus Brevis Tear with Autologous Gracilis: A Case Report
}

\author{
Gautam Kumar ${ }^{1}$, Dennis P Jose ${ }^{2}$, Rajesh Simon ${ }^{3}$, Surekh Ravi ${ }^{4}$, Deepu J Punnoose ${ }^{5}$
}

\begin{abstract}
Aim: Gracilis autograft repair for an isolated atraumatic peroneus brevis rupture.

Background: Peroneal tendon injuries are one of the causes of lateral ankle pain and instability that can either involve peroneus brevis tendon (PBT) or peroneus longus tendon (PLT). PBT tears are often associated with trauma, leading to forced dorsiflexion of ankle or chronic subluxation. Atraumatic tear of PBT is rare, with only a few cases described. The surgical management for PBT tear includes peroneal tendoscopy, debridement and tubulization of remaining tendon and tenodesis. There is no consensus regarding the optimal surgical intervention for irreparable tears.

Case description: A 43-year-old businessman presented to our clinic with complaints of pain and swelling in lateral side of left ankle of 1 week duration. The clinical examination revealed high-arched foot with diffuse swelling over the retrofibular area with tenderness elicited along the line of PBT. The sagittal view of ankle MRI showed completed tear of PBT. A curvilinear lateral incision was made along the posterior border of fibula. The proximal end of donor graft was secured to the proximal end of remaining PBT using pulverate weaving technique, while the distal end graft was looped through the drill hole in 5th metatarsal base and sutured to itself.

Conclusion: Autograft is biologically superior to allograft with the advantages of tissue compatibility and faster reincorporation.

Clinical significance: Surgical management of PBT tears are advocated for active, symptomatic, or patients who fail to improve with conservative management.

Keywords: Ankle joint, Gracilis autograft, Peroneal, Peroneus brevis, Peroneus brevis tear, Pulverate weaving technique, Tendon repair. Journal of Foot and Ankle Surgery (Asia Pacific) (2022): 10.5005/jp-journals-10040-1200
\end{abstract}

\section{BACKGROUND}

Ankle sprains are among the commonest soft tissue injuries with an incidence of 1 per 10,000 daily ${ }^{1}$ and account for nearly one-fifth of exercise-related injuries. ${ }^{2}$ Ankle instability creates a vicious circle of repetitive sprains and recurrent injuries. Ankle injuries can affect both medial and lateral ligaments, but in nearly $85 \%$ of cases, the lateral side ligaments are affected. ${ }^{2}$ Peroneal tendon injuries are one of the causes of lateral ankle pain and instability that can either involve the peroneus brevis tendon (PBT) or the peroneus longus tendon (PLT). The underlying pathophysiology involves a combination of mechanical and anatomical factors that may include tendinopathy, longitudinal splits, partial or complete tears. ${ }^{3}$ Peroneus brevis tendon tears are often associated with an acute trauma leading to forced dorsiflexion of the ankle or chronic subluxation leading to torn superior retinaculum or misalignment fibula. Atraumatic tear of PBT is rare, ${ }^{4}$ with only a few cases described.

The conservative management includes ankle immobilizers, protected weight-bearing, and analgesics. The surgical management for PBT tear includes peroneal tendoscopy, debridement, and tubulization of the remaining tendon and tenodesis. Operative treatment for irreparable tears includes flexor hallucis longus (FHL) or flexor digitorum longus (FDL) tendon transfers. There is still no consensus regarding the optimal surgical intervention while using autografts for reconstruction. We present a case report of gracilis autograft for an isolated peroneus brevis rupture, which has not been described for this indication to the best of our knowledge.

\section{Case Description}

A 43-year-old businessman presented to our clinic with complaints of pain and swelling in the lateral side of the left ankle of a
${ }^{1-5}$ Department of Orthopedics, VPS Lakeshore Hospital, Kochi, Kerala, India

Corresponding Author: Rajesh Simon, Department of Orthopedics, VPS Lakeshore Hospital, Kochi, Kerala, India, Phone: +91 9447634466, e-mail: footandanklekochi@gmail.com

How to cite this article: Kumar G, Jose DP, Simon R, et al. Repair of Peroneus Brevis Tear with Autologous Gracilis: A Case Report. J Foot Ankle Surg (Asia Pacific) 2022;9(1):25-29.

Source of support: Nil

Conflict of interest: None

1-week duration. The pain sudden in the onset of dull aching type, aggravated on exertion and walking on uneven surfaces, with a visua analog score (VAS) of 6 . The patient gave the history of a snapping sound, heard while on a morning walk, with no significant history of trauma. There was no rest pain or night pain. On examination, there was a high-arched foot with diffuse swelling over the retrofibular area with tenderness elicited along the line of PBT. The ankle movements were pain-free except for inversion. The preoperative foot and ankle disability score (FADI) ${ }^{5}$ was 63.5 out of a maximum of 104. The routine blood examination and infection markers, including total leukocyte count (TLC), erythrocyte sedimentation rate (ESR), and C-reactive protein (CRP), were within normal limits. The weight-bearing X-rays of the ankle in anteroposterior (AP), lateral, and mortise view (Fig. 1) showed a high-arched foot with no obvious fracture.

The patient was advised ankle MRI. The sagittal view of ankle MRI showed a completed tear of the peroneus brevis with a gap of around $5.8 \mathrm{~cm}$ (Fig. 2). The axial view of ankle MRI shows the 

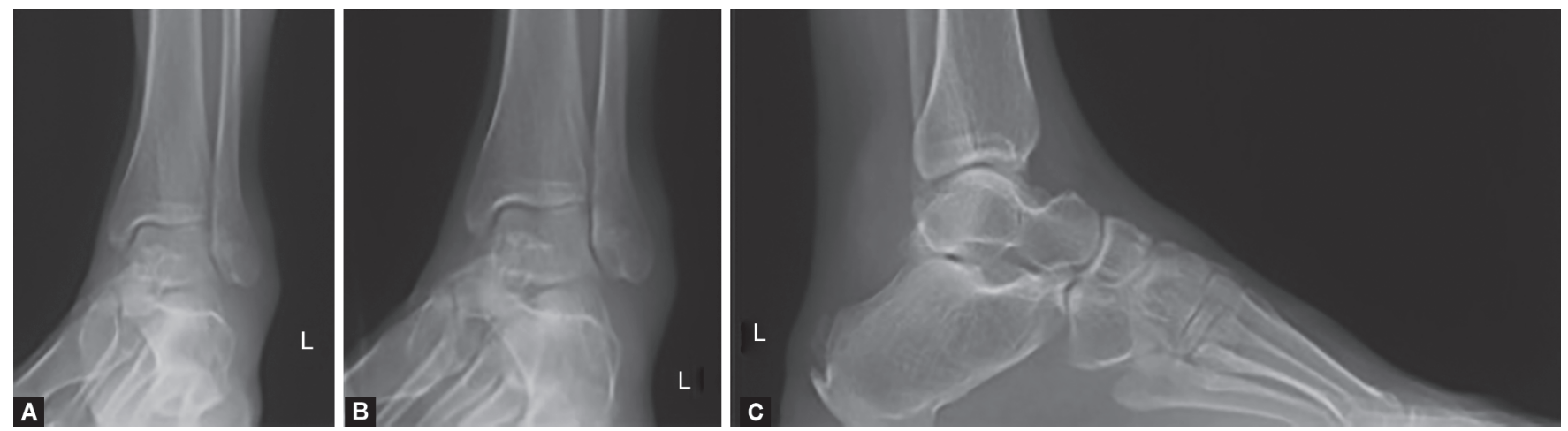

Figs $1 \mathrm{~A}$ to $\mathrm{C}$ : The anteroposterior (AP), mortise, and lateral view of X-ray showing high-arch foot

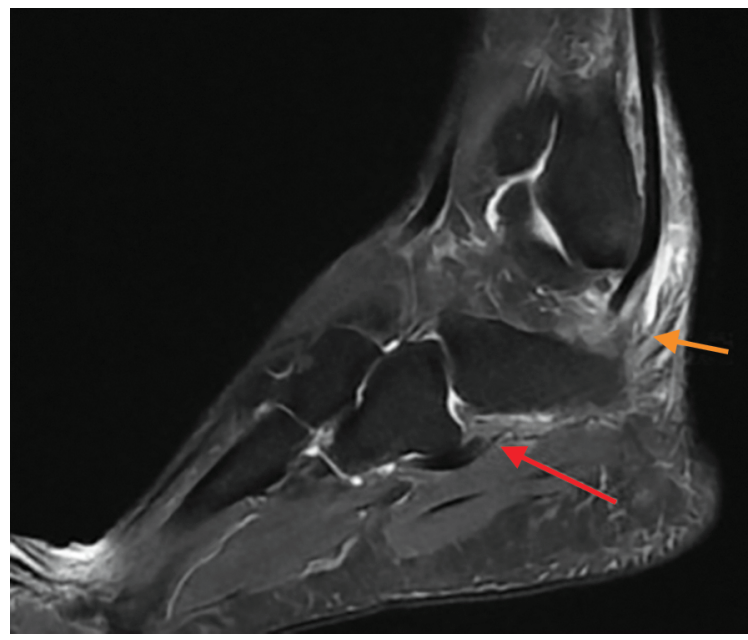

Fig. 2: Sagittal proton dense fat-suppressed (PDFS) image with red arrow showing the distal stump while orange arrow depicts the empty tendon sheath of the peroneus brevis tendon (PBT)

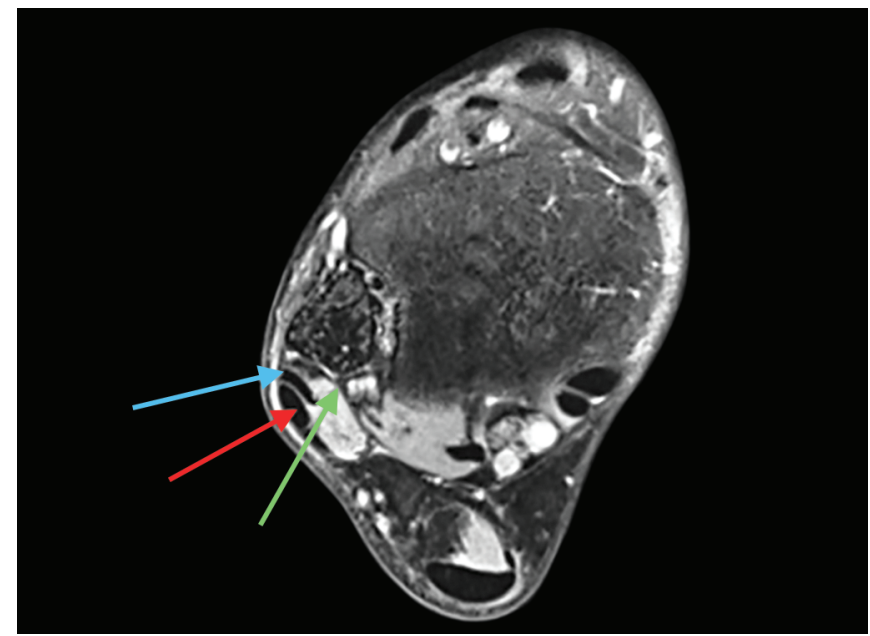

Fig. 4: Axial proton dense fat-suppressed (PDFS) image with a blue arrow showing the normal semicircular peroneus brevis tendon (PBT), red arrow representing the peroneus longus tendon (PLT) while green arrow points to the tendon sheath in normal subjects

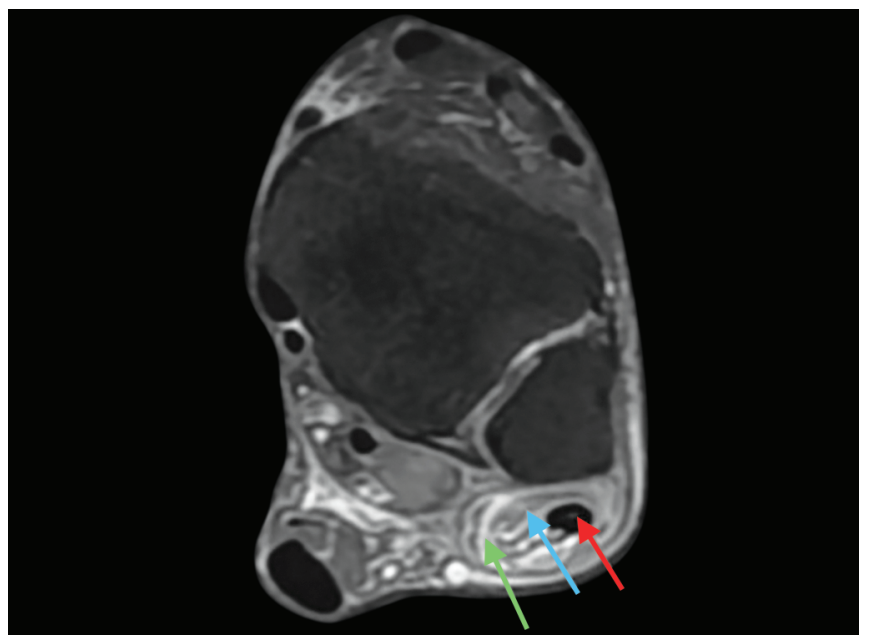

Fig. 3: Axial proton dense fat-suppressed (PDFS) image with a blue arrow showing the ghost/empty peroneus brevis tendon (PBT), red arrow representing the peroneus longus tendon (PLT) while green arrow points to the tendon sheath in the patient

ghost tendon of PBT (Fig. 3) compared with the PBT in a normal patient (Fig. 4).

The patient was given a choice for a bony procedure for the correction of the high-arch foot deformity along with the repair of PBT, but the patient opted only for PBT repair.

\section{Surgical Technique}

Position

The patient was positioned in a lazy lateral position.

\section{Incision}

A curvilinear lateral incision of around $7 \mathrm{~cm}$ was made, beginning $4 \mathrm{~cm}$ proximal from the tip of fibula extending up to the base of the 5th metatarsal along the posterior border of the fibula (Fig. 5).

\section{Finding}

The superior peroneal retinaculum (SPR) was incised to expose the peroneal tendons safeguarding the sural nerve. The peroneal sheath opened up. PL tendon was intact. Complete rupture of PBT with retracted ends and defect of approximately $6 \mathrm{~cm}$ (Fig. 6) was 


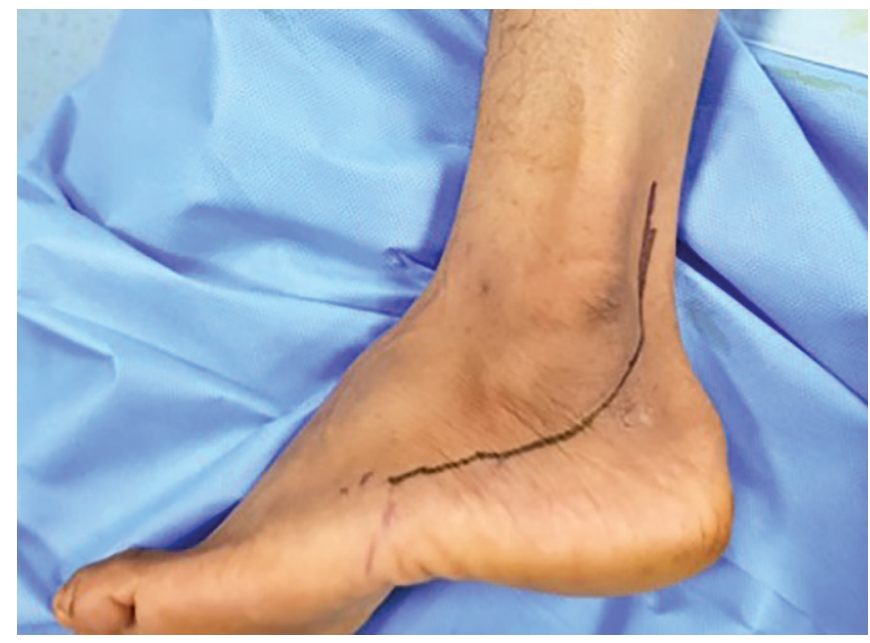

Fig. 5: The curvilinear lateral incision along the posterior border of fibula extending up to the base of the 5 th metatarsal

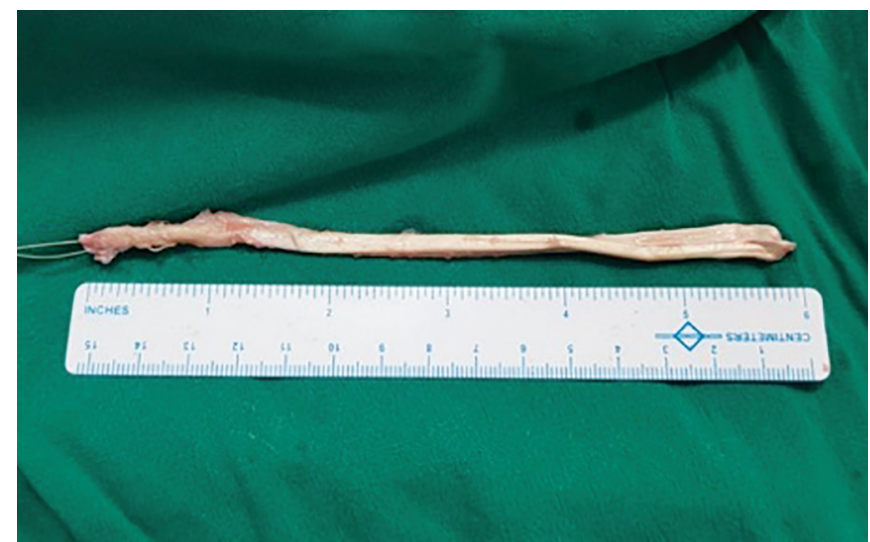

Fig. 7: The harvested autologous gracilis tendon with side-to-side tenodesis done with 1-0 Vicryl

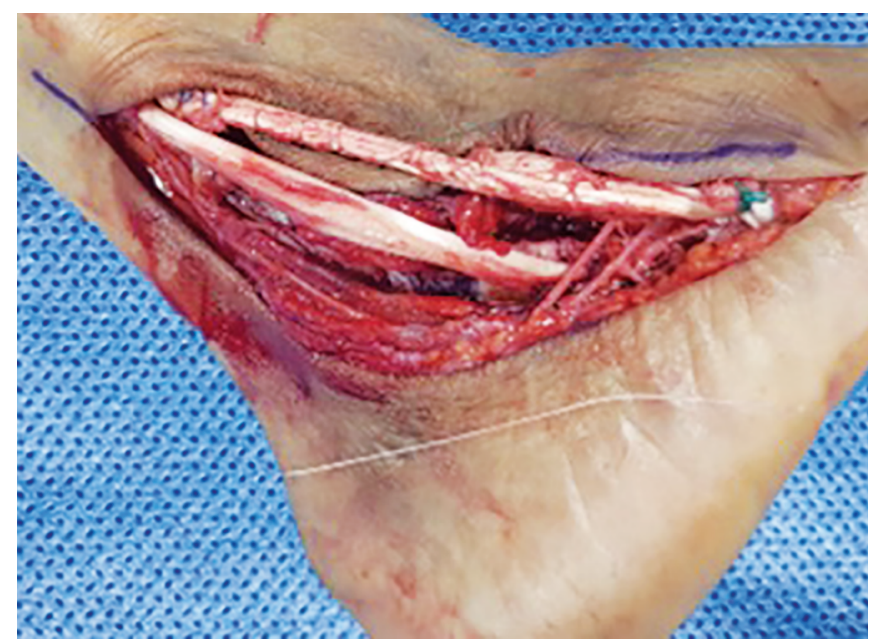

Fig. 9: The defect in the PBT was filled with gracilis graft where the proximal end was secured to the proximal end of the original remaining PBT using pulverate weaving technique while the distal end looped through the 5th MT base

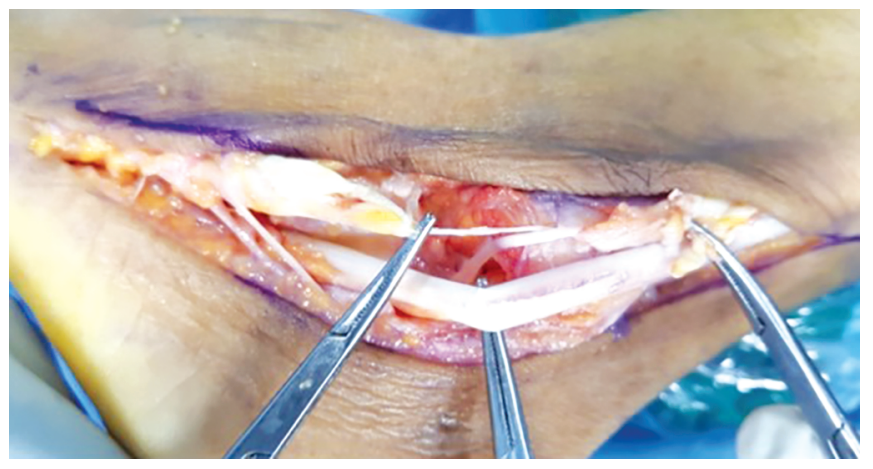

Fig. 6: The retromalleolar gap in the PBT due to complete rupture of PBT with retracted ends

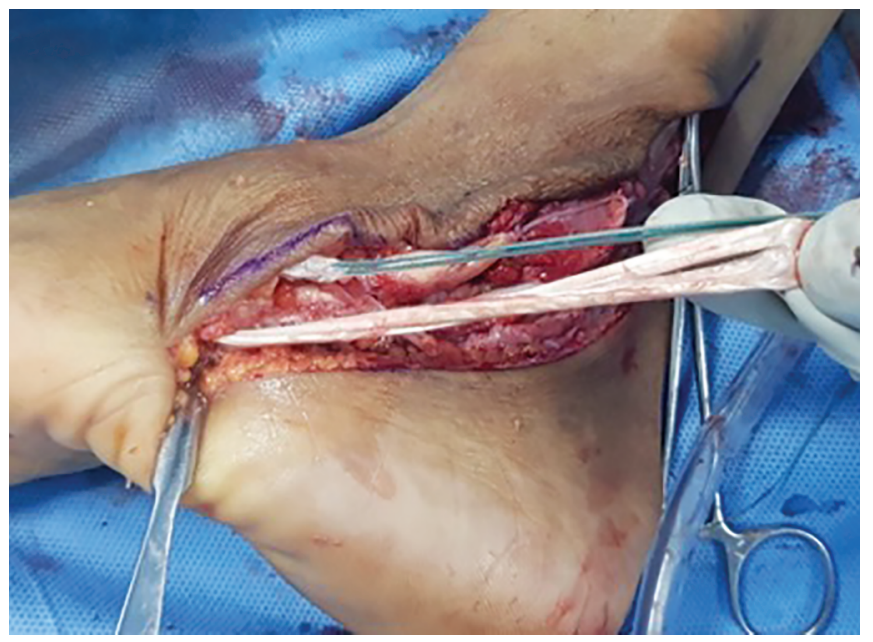

Fig. 8: The gracilis graft passed through the drill hole in the base of the 5th metatarsal (MT) and sutured to itself

noted around the distal end of the fibula. The three lateral ankle ligaments, anterior talofibular ligament (ATFL), calcaneofibular ligament (CFL), and posterior talofibular ligament (PTFL), were intact without any laxity.

\section{Graft Preparation}

A 3-cm medial longitudinal incision was placed at the proximal tibia. The gracilis was identified and resected. The graft was doubled, and side to side tenodesis was done with 1-0 Vicryl (Fig. 7).

\section{Procedure}

The tethered portion of the tendon was debrided and removed, leaving a 7-cm gap between the two ends of the tendon. A hole was drilled in the base of the 5th MT from plantar to dorsal aspect using a 4-mm drill bit. The graft was looped through the drill hole and sutured to itself (Fig. 8). The distal stump of PBT was sutured to the distal end of the graft. The proximal end of the donor graft was secured to the proximal end of PBT (Fig. 9) using pulverate weaving technique, and the tone of the tendon graft was found satisfactory while keeping the foot in the neutral position. Superior peroneal retinaculum was then repaired (Fig. 10), and the wound was closed in layers. 


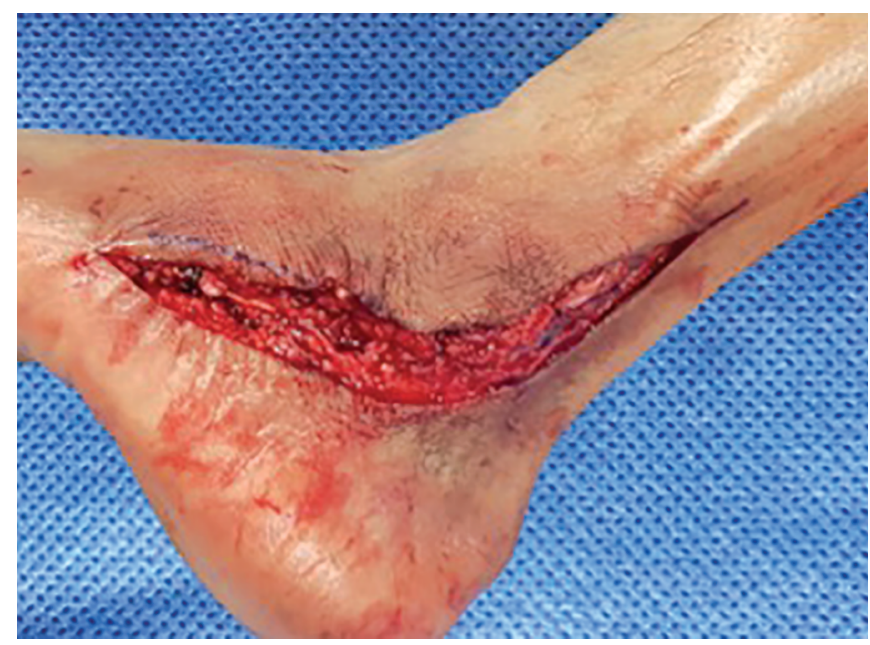

Fig. 10: The repaired superior peroneal retinaculum (SPR) layer before skin closure

\section{Postoperative Management}

The patient was put on a below-knee slab. After 2 weeks, sutures were removed. Weight-bearing was delayed till 6 weeks, following which assisted weight-bearing was started. By 8 weeks, he progressed to full weight-bearing without support. The rehabilitation program started to improve the range of motion and strength. After 3 months, the patient resumed his physical activities and was pain-free both in the foot and the donor site. Six months following the surgery, the patient had excellent clinical outcomes. The FADI score improved to 93.3 , while VAS for pain decreased to 1 .

\section{Discussion}

The initial assessment of acute ankle injury should follow the Ottawa ankle guidelines. The initial management of the acute injury is RICE (Rest, Ice, Compression, and Elevation). The US and MRI are appropriate modalities for the evaluation of peroneal tendons. The prevalence of peroneal tears in the general population is unknown, but cadaveric studies have pointed out the incidence to be as high as $38 \%{ }^{6}$

Peroneal tendon injuries are often classified as acute or chronic, the description being more related to the mechanism of injury rather than duration. Peroneus brevis tendon tears are more common than PLT. Isolated atraumatic rupture of PBT is a rare occurrence. A low-lying muscle belly, accessory peroneal muscles (peroneus quadratus and peroneus quintus), convex or flat fibular groove, the integrity of SPR, and a cavovarus foot type are some of the anatomical factors predisposing to a peroneal tendon injury.

The commonest pattern of tendon tear is longitudinal, centered at the level of the tip of the fibula in the region of the peroneal sulcus. There is increased mechanical stress on the tendon as it changes its direction sharply in this region. Compression of the brevis tendon between the longus and fibula also adds to the mechanical stress and can lead to fraying or longitudinal splitting of the tendon in this location. The PBT has a relative avascular zone as it passes through the retrofibular groove. Though there are multiple reports on longitudinal splits in PBT, only a few mentions isolated full-thickness PBT rupture.
Conservative management of isolated PBT injury includes immobilization in a cast in plantarflexion or boot with a heel raise that can be tried in low demand and asymptomatic patients. Conservative treatments for PBT tears have shown poor clinical outcomes with a failure rate of $50-83 \%,{ }^{7}$ necessitating surgical intervention. Surgical management is advocated for active, symptomatic, or failed conservative patients. The additional procedure of SPR and retromalleolar groove deepening can be employed along with the repair.

The $50 \%$ cross-sectional area threshold, as advocated by some authors, ${ }^{8}$ is arbitrary, as it is not based on good quality data. The primary debridement and repair can be tried even if $<50 \%$ cross-sectional area of reasonable native tendon is left based on the surgeon's assessment. For irreparable tears of the tendon, the options include segmental resection followed by tenodesis, tendon transfer, or reconstruction using allograft or autograft.

Redfern and Myerson ${ }^{8}$ recommended that if an irreparable tear is present in one tendon and the other is healthy, tenodesis should be performed, but the biomechanical study by Pellegrini et al. ${ }^{9}$ concluded that tenodesis to the PLT did not effectively restore peroneus brevis tension and sacrifices the integrity of the muscle-tendon unit. A second option is the deep flexor tendon transfers, but these procedures also have biomechanical limitations with the unsatisfactory restoration of strength and eversion power. ${ }^{10}$

Reconstruction with tendon allograft has shown excellent clinical outcomes and restoration of tendon function without sacrificing any adjacent structure or adding morbidity. Concerns for allograft use include potential disease transmission, risk of an immune response, delayed incorporation of the graft at the recipient site, and stretching of the graft (creep). ${ }^{11}$

Literature regarding using autograft for $\mathrm{PBT}$ reconstruction is scarce. There is concern regarding the donor site morbidity but, an autograft is biologically superior to allograft with advantages of tissue compatibility and faster reincorporation. Nishikawa et al. ${ }^{12}$ reported satisfactory clinical results using semitendinosus autograft. To the best of our knowledge, this is the first report of using gracilis autograft for isolated peroneus brevis rupture. Our patient did not have any residual pain at the donor site and resumed pre-injury level activities after 6 months without any gait alteration.

\section{ConClusion}

Peroneal tendon injuries are common, especially peroneal brevis. However, an atraumatic complete tear of the peroneal brevis in the high-arched foot has to be taken into consideration while assessing the ankle pain. The US and MRI are appropriate modalities for the evaluation of peroneal tendons to support the clinical diagnosis. There is no consensus regarding the optimal surgical intervention for irreparable tears.

\section{Clinical Significance}

Surgical management is advocated for active, symptomatic, or failed conservative patients. Autograft is biologically superior to allograft with the advantages of tissue compatibility and faster reincorporation. To the best of our knowledge, this is the first report of using gracilis autograft for isolated peroneus brevis rupture. 


\section{References}

1. Chauhan B, Panchal P, Szabo E, et al. Split peroneus brevis tendon: an unusual cause of ankle pain and instability. J Am Board Fam Med 2014;27(2):297-302. DOI: 10.3122/jabfm.2014.02.130009.

2. Polzer H, Kanz KG, Prall WC, et al. Diagnosis and treatment of acute ankle injuries: development of an evidence-based algorithm. Orthop Rev 2012;4(1):e5. DOI: 10.4081/or.2012.e5.

3. Heckman DS, Gluck GS, Parekh SG. Tendon disorders of the foot and ankle, part 1: peroneal tendon disorders. Am J Sports Med 2009;37(3):614-625. DOI: 10.1177/0363546508331206.

4. Minoyama $\mathrm{O}$, Uchiyama $\mathrm{E}$, Iwaso $\mathrm{H}$, et al. Two cases of peroneus brevis tendon tear. Br J Sports Med 2002;36(1):65-66. DOI: 10.1136/ bjsm.36.1.65.

5. Hale SA, Hertel J. Reliability and sensitivity of the Foot and Ankle Disability Index in subjects with chronic ankle instability. J Athlet Train 2005;40(1):35.

6. Sobel M, Bohne WH, Levy ME. Longitudinal attrition of the peroneus brevis tendon in the fibular groove: an anatomic study. Foot Ankle 1990;11(3):124-128. DOI: 10.1177/107110079001100302.
7. McLennan JG. Treatment of acute and chronic luxations of the peroneal tendons. Am J Sports Med 1980;8(6):432-436. DOI: $10.1177 / 036354658000800609$.

8. Redfern D, Myerson M. The management of concomitant tears of the peroneus longus and brevis tendons. Foot Ankle Int 2004;25(10):695707. DOI: $10.1177 / 107110070402501002$.

9. Pellegrini MJ, Glisson RR, Matsumoto T, et al. Effectiveness of allograft reconstruction vs tenodesis for irreparable peroneus brevis tears: a cadaveric model. Foot Ankle Int 2016;37(8):803-808. DOI: 10.1177/1071100716658469.

10. Seybold JD, Campbell JT, Jeng CL, et al. Outcome of lateral transfer of the FHL or FDL for concomitant peroneal tendon tears. Foot Ankle Int 2016;37(6):576-581. DOI: 10.1177/1071100716634762.

11. Strickland SM, MacGillivray JD, Warren RF. Anterior cruciate ligament reconstruction with allograft tendons. Orthop Clin North Am 2003;34(1):41-47. DOI: 10.1016/s0030-5898(02)00060-3.

12. Nishikawa DR, Duarte FA, Saito GH, et al. Reconstruction of the peroneus brevis tendon tears with semitendinosus tendon autograft. Case Rep Orthop 2019;2019:5014687. DOI: 10.1155/2019/5014687. 